\title{
Establishment and characterization of a human adenoid cystic carcinoma cell line forming colonies cultured in collagen gel and transplantable in nude mice
}

\author{
NORIAKI TANAKA ${ }^{1}$, KAZUHIKO URABE ${ }^{2}$, SUSUMU HASHITANI ${ }^{1}$, \\ KAZUNARI SAKURAI ${ }^{1}$ and MASAHIRO URADE ${ }^{1}$
}

\begin{abstract}
${ }^{1}$ Department of Oral and Maxillofacial Surgery, Hyogo College of Medicine, 1-1 Mukogawa-cho, Nishinomiya, Hyogo 663-8501;
${ }^{2}$ Dentistry and Oral Surgery, Yao Tokushukai General Hospital, 3-15-38 Kyuhoji, Yao, Osaka 581-0072, Japan
\end{abstract}

Received September 26, 2006; Accepted October 30, 2006

\begin{abstract}
We established a new cell line (ACCNS) from human adenoid cystic carcinoma (AdCC) of the maxilla, using tissue culture techniques and it has been successfully subcultured for more than 100 passages during 2 years. The population doubling time was approximately $39.2 \mathrm{~h}$ on a plastic dish. In type I collagen gel culture, the cells formed spherical colonies by day 7 after seeding. The colonies showed tubular and solid structures, and eosinophilic material stained with mucicarmine was revealed in the inner space. Immunohistochemically, ACCNS cells demonstrated expressions of keratin, $\alpha$-smooth muscle actin, vimentin and S-100 protein, similar to those of original AdCC. These findings indicate that ACCNS cells possess the characteristics of AdCC. In addition, inoculation of $4.0 \times 10^{6}$ cells into nude mice developed tumors that were histologically confirmed as undifferentiated carcinoma. Therefore, ACCNS is the first AdCC cell line with tumorigenicity in nude mice. Based on these results, ACCNS provides a useful culture model of AdCC to analyze the biological characteristics and behavior of this tumor.
\end{abstract}

\section{Introduction}

AdCC is a common malignant tumor of the salivary gland (1) and characterized by slow-growing, diffuse invasion into adjacent tissues and high incidence of distant metastasis (2). It is difficult to cure patients with this tumor because of its characteristics. Therefore, it is essential to establish AdCC cell line to analyze its biologic behavior. Two AdCC tumor lines have been established transplantable in nude mice $(3,4)$, and

Correspondence to: Dr Noriaki Tanaka, Department of Oral and Maxillofacial Surgery, Hyogo College of Medicine, 1-1 Mukogawa-cho, Nishinomiya, Hyogo 663-8501, Japan

E-mail: ntanaka@hyo-med.ac.jp

Key words: adenoid cystic carcinoma cell line, three-dimensinal culture, colony formation, tumorigenicity in nude mice only one study has reported an AdCC cell line cultured in three-dimensional condition (5). However, there is no available literature concerning establishment of AdCC cell line with tumorigenicity in nude mice. We have successfully established a human AdCC cell line capable of being cultured in three-dimensional condition of type I collagen gels. This cell line showed a monolayer culture on plastic dishes, and formed spherical colonies in type I collagen gel culture. Furthermore, inoculation of cells into nude mice produced tumors showing undifferentiated carcinoma histologically. In this study, we report establishment and characterization of a new AdCC cell line.

\section{Materials and methods}

Establishment of a cell line. Tumor tissue was obtained from the surgical material of a 59-year-old male patient with tubular and cribriform patterns of AdCC of the maxilla (Fig. 1A). The tissue was rinsed twice with sterile $\mathrm{Ca}^{2+}$ and $\mathrm{Mg}^{2+}$-free phosphate-buffered saline (PBS) containing 1000 units/ml of penicillin and cut with scissors into pieces of 1-2 mm, then placed into a $100-\mathrm{mm}$ plastic culture dish and cultured in Dulbecco's modified Eagle's medium (DMEM, Nissui Pharmaceutical Co., Ltd., Tokyo, Japan) supplemented with $10 \%$ fetal bovine serum (FBS, HyClone Laboratories, UT, USA) and $2 \mathrm{mM}$ L-glutamine (Wako Pure Chemical Industries Ltd., Osaka, Japan) as growth medium at $37^{\circ} \mathrm{C}$ in a $5 \% \mathrm{CO}_{2}$ incubator. Approximately, 10 days later, outgrowth of cells from the explant was observed and most of them consisted of a mixture of epithelioid cells, myoepithelioid cells and fibroblasts. The cell mixture was cultured on feeder layer of fibroblasts to remove fibroblasts from cell mixture. By culturing on feeder layer twice, fibroblasts were completely removed. When the epithelioid and myoepithelioid cells reached subconfluency, they were subcultured after dissociation with $0.25 \%$ trypsin and $1 \mathrm{mM}$ EDTA mixture and grown on 100-mm plastic culture dishes for the following studies.

Cell growth assay. The cells were plated at the density of $5.0 \times 10^{3}$ cells/well onto 96-well tissue culture plates (Iwaki Glass Co., Ltd., Funabashi, Japan) and incubated at $37^{\circ} \mathrm{C}$ in 


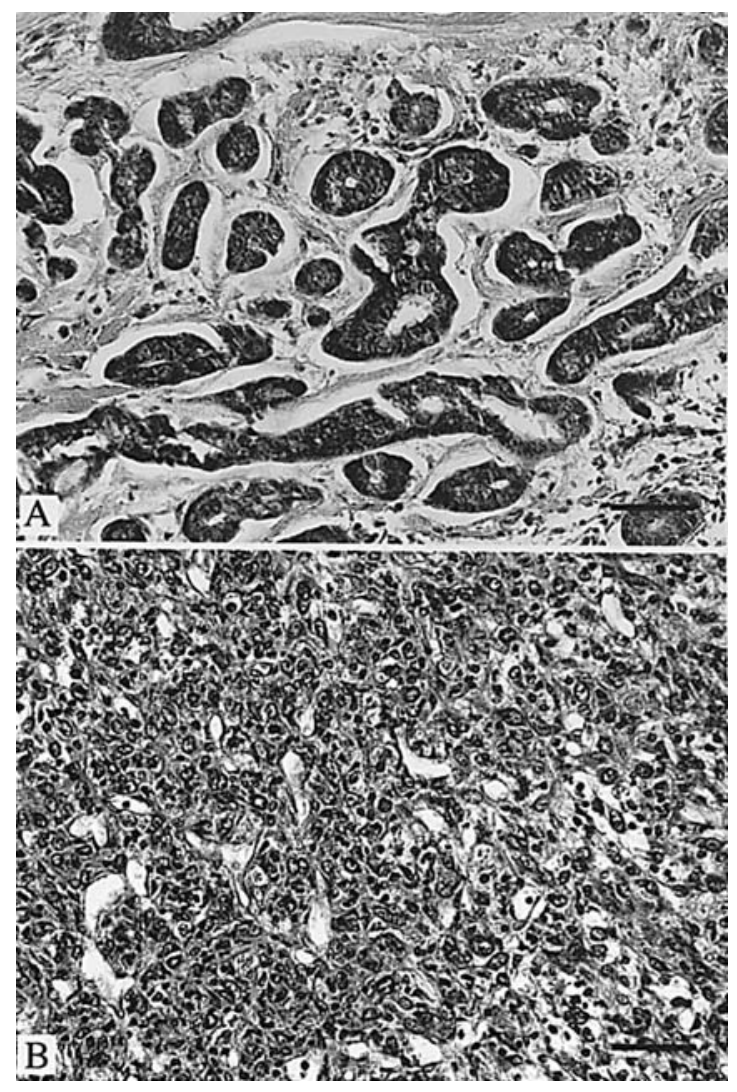

Figure 1. Histological features of the original tumor and the tumor produced in nude mouse. (A), Original AdCC arising in the maxilla showing tubular pattern; (B), nude mouse tumor showing undifferentiated carcinoma consisted of a diffuse, non-cohesive proliferation of polyhedral epithelial cells with oval or round vesicular nuclei, prominent epsinophilic nucleoli, scant cytoplasm and indistinct cell margins (H\&E stain; bar, $100 \mu \mathrm{m}$ ).

a $5 \% \mathrm{CO}_{2}$ incubator. The viable cells were counted at $24 \mathrm{~h}$ intervals by using a dye exclusion test with nigrosin in triplicate wells. The population doubling time was calculated from the resulting growth curve.

Two dimensional/three dimensional collagen gel cultures. Two dimensional (2D) or three dimensional (3D) culture was performed by using the modified procedure by Yang et al (6). Briefly, type I collagen extracted from the porcine achilles tendon (Cellmatrix type I-A, Nitta Gelatin Inc., Osaka, Japan) was used as matrix. The collagen gel culture with physiological ionic strength, $\mathrm{pH} 7.4$, was made by mixing seven volume of $0.3 \%$ iced-cold type I collagen in $0.001 \mathrm{~N} \mathrm{HCl}$, two volume of 5-fold strength DMEM solution and one volume of reconstruction buffer $\left(2.2 \mathrm{~g}\right.$ of $\mathrm{NaHCO}_{3}$ and $200 \mathrm{mM}$ of HEPES in $100 \mathrm{ml}$ of $0.05 \mathrm{~N} \mathrm{NaOH}$ ). A volume of $100 \mu 1$ for $2 \mathrm{D}$ cultures or $50 \mu \mathrm{l}$ for $3 \mathrm{D}$ cultures diluted collagen gel (type I collagen gel and culture medium were mixed at equal volume) was added to 96-well tissue culture plates and incubated at $37^{\circ} \mathrm{C}$ for $30 \mathrm{~min}$ to form gel as basal layer. For $2 \mathrm{D}$ cultures, $2.5 \times 10^{3}$ ACCNS cells were added onto basal layer in $100 \mu 1$ of culture medium. For $3 \mathrm{D}$ cultures, $2.5 \times 10^{3}$ ACCNS cells were added into $50 \mu 1$ collagen gel, which was kept on ice to prevent gelling, and overlaid on a basal layer in 96-well plastic dishes and incubated at $37^{\circ} \mathrm{C}$ for $30 \mathrm{~min}$ to form a gel embedded with ACCNS cells. Then, the gel was overlaid with $100 \mu 1$ of culture medium. The medium was changed every day. The cells on or in the gel were cultured for 3 weeks. The growth and shape of ACCNS cells were observed and photographed with a phase-contrast microscope (Leica, Wetzlar, Germany).

Inoculation of cultured cells into nude mice. The cultured cells were trypsinized, washed twice and suspended in PBS. Then $4.0 \times 10^{6}$ cells in $0.2 \mathrm{ml}$ PBS were inoculated subcutaneously into the flanks of 5-week-old female nude mice (BALB/c nu/nu, Charles River Japan, Yokohama, Japan). The animals were fed ad libitum under sterile conditions during the experimental period. When tumors developed at the inoculated sites, mice were sacrificed and tumors were removed for histological and immunohistochemical examinations. All animal experiments were performed in compliance with the Guidelines for Animal Experiments of Hyogo College of Medicine.

Histological examination. ACCNS cell colonies in collagen gels and nude mouse tumors were fixed with Carnoy's fluid $(\mathrm{MeOH}: \mathrm{AcOH}=3: 1)$ for $3 \mathrm{~h}$ at room temperature. They were dehydrated and embedded in paraffin. Serial $4 \mu \mathrm{m}$ sections were cut and mounted on glass slide. Deparaffinized sections were stained with hematoxylin and eosin (H\&E) and mucicarmine (mucicarmine stain: Mayer's mucicarmine solution, Sigma). These sections were observed and photographed with light microscope (Olympus, Tokyo, Japan).

Immunohistochemical examination. For immunohistochemical examination, a modified streptavidin-biotin-peroxidase complex (SABC) method was employed (7). The thin sections of ACCNS cell colonies in collagen gels and original tumor of the patient were deparaffinized and rehydrated in a graded series of alcohols. The samples were incubated at $95^{\circ} \mathrm{C}$ for $60 \mathrm{~min}$ in citrate buffer ( $\mathrm{pH} \mathrm{6.0)}$ ) for antigen retrieval. Endogenous peroxidase activity was blocked with $3 \% \mathrm{H}_{2} \mathrm{O}_{2}$ in methanol for $15 \mathrm{~min}$. After washing with PBS, the samples were treated with $10 \%$ normal goat serum in PBS for $30 \mathrm{~min}$ to block non-specific background staining. Monoclonal antibodies against $\alpha$-smooth muscle actin ( $\alpha$-SMA, Sigma, MO, USA, 1:1000), epithelial membrane antigen (EMA, YLEM, Rome, Italy, 1:300) and vimentin (Sigma, 1:300) and rabbit polyclonal antibody against human keratin (A575: Dako, Glostrup, Demark, 1:1000) and human S-100 protein (Dako, 1:1000) were applied as primary antibodies at $4^{\circ} \mathrm{C}$ for $18 \mathrm{~h}$. After washing with PBS, biotinylated goat anti-mouse IgG and anti-rabbit IgG mixture (Histofine SAB-PO kit, Nichirei Co., Ltd., Tokyo, Japan) was applied to the sections, and incubated for $10 \mathrm{~min}$ at room temperature. After further washing with PBS, the sections were incubated with the peroxidase labeled streptavidin solution at room temperature for $5 \mathrm{~min}$. Peroxidase reactivity was visualized with Histofine DAB substrate kit (Nichirei). Finally, the sections were slightly counterstained with hematoxylin. These immunohistochemical sections were observed and photographed with light microscope (Olympus).

Immunofluorescence examination. The 2D or 3D cultured ACCNS cells were examined by immunofluorescence staining. 
These samples were prepared on chamber slide II (Iwaki Glass Co.). For 2D cultures, 1.0x $10^{4}$ ACCNS cells were added to each chamber in $200 \mu \mathrm{l}$ of culture medium. For 3D cultures, $100 \mu 1$ of diluted collagen gel including $2.5 \times 10^{3}$ ACCNS cells was added to each chamber and incubated at $37^{\circ} \mathrm{C}$ for $30 \mathrm{~min}$. Then, the gel was overlaid with $100 \mu \mathrm{l}$ of culture medium. The medium of both samples was changed every day. After one week in $2 \mathrm{D}$ cultures or 2 weeks in $3 \mathrm{D}$ cultures, these samples were washed with PBS twice, and fixed with $3.7 \%$ formaldehyde solution in PBS for $10 \mathrm{~min}$ at room temperature. After washing with PBS, the samples were treated with $0.1 \%$ Triton-X 100 (Wako) in PBS for 5 min. After washing with PBS, the samples were treated with $1 \%$ bovine serum albumin (BSA: Wako Pure Chemical Industries, Ltd.), 0.03\% Tween-20 (Wako Pure Chemical Industries, Ltd.) in PBS for 30 min to block non-specific background staining. Monoclonal antibodies against human $\alpha$-SMA, EMA and vimentin and rabbit polyclonal antibody against human keratin and S-100 protein were applied as primary antibodies at $4^{\circ} \mathrm{C}$ for $18 \mathrm{~h}$. After further washing with PBS, FITC-labeled goat anti-mouse IgG or anti-rabbit IgG antibody (Molecular Probes Inc., OR, USA, 1:500) was applied to the samples, and incubated for $60 \mathrm{~min}$ at room temperature. After washing with PBS, Rhodaminered-labeled phallotoxin (Molecular Probes, 1:400) was applied to the samples, and incubated for $20 \mathrm{~min}$ at room temperature. After further washing with PBS, the samples were treated with $10 \mathrm{nM}$ DAPI (Molecular Probes) for $5 \mathrm{~min}$ at room temperature. Finally, the samples were mounted with Prolong Antifade Kit (Molecular Probes). The 3D samples were treated with $3.7 \%$ formaldehyde in PBS, $0.1 \%$ Triton-X 100 in PBS, Rhodamin-red-labeled phallotoxin and $10 \mathrm{nM}$ DAPI in the same manner. These immunofluorescence samples were observed and photographed by LSM510 of laser scanning microscope (Carl Zeiss Co., Ltd., Oberkochen, Germany), and analyzed with Image Browser (Carl Zeiss).

RT-PCR analysis. Total-RNAs from ACCNS, HSG (human adenocarcinoma cell line) (8), SCC25 (human tongue carcinoma cell line) (9) and nude mouse tumor were extracted using RNeasy MinElute Cleanup Kit (Qiagen, Hilden, Germany) and reverse transcribed by First-Strand cDNA Synthesis Kit (Amersham Biosciences, Buckinghamshire, UK). The resultant cDNAs were used as the template for PCR using primer specific to human $B$-actin as follow: sense, 5'-TGACGGGGTCACCCACACTGTGCCCATCTA-3' and antisense, 5'-CTAGAAGCATTTGCGGTGGACGATGGA GGG-3'. PCR was done with denaturation at $94^{\circ} \mathrm{C}$ for $5 \mathrm{~min}$, then 35 cycles at $94^{\circ} \mathrm{C}$ for $30 \mathrm{sec}$, at $66^{\circ} \mathrm{C}$ for $30 \mathrm{sec}$, and at $72^{\circ} \mathrm{C}$ for $1 \mathrm{~min}$, and then at $72^{\circ} \mathrm{C}$ for $7 \mathrm{~min}$. PCR products were electrophoresed in $1.5 \%$ agarose gels, stained with ethidium bromide and visualized under UV illumination.

\section{Results}

Establishment of a new AdCC cell line. The newly established cell line designated as ACCNS has been subcultured more than 100 passages during 2 years. ACCNS cells showed a monolayer culture on a plastic dish, consisting of small cuboidal cells and large spherical cells. Small cuboidal cells showed flattened epithelioid shape with occasional slender processes (Fig. 2A), and large spherical cells contained many

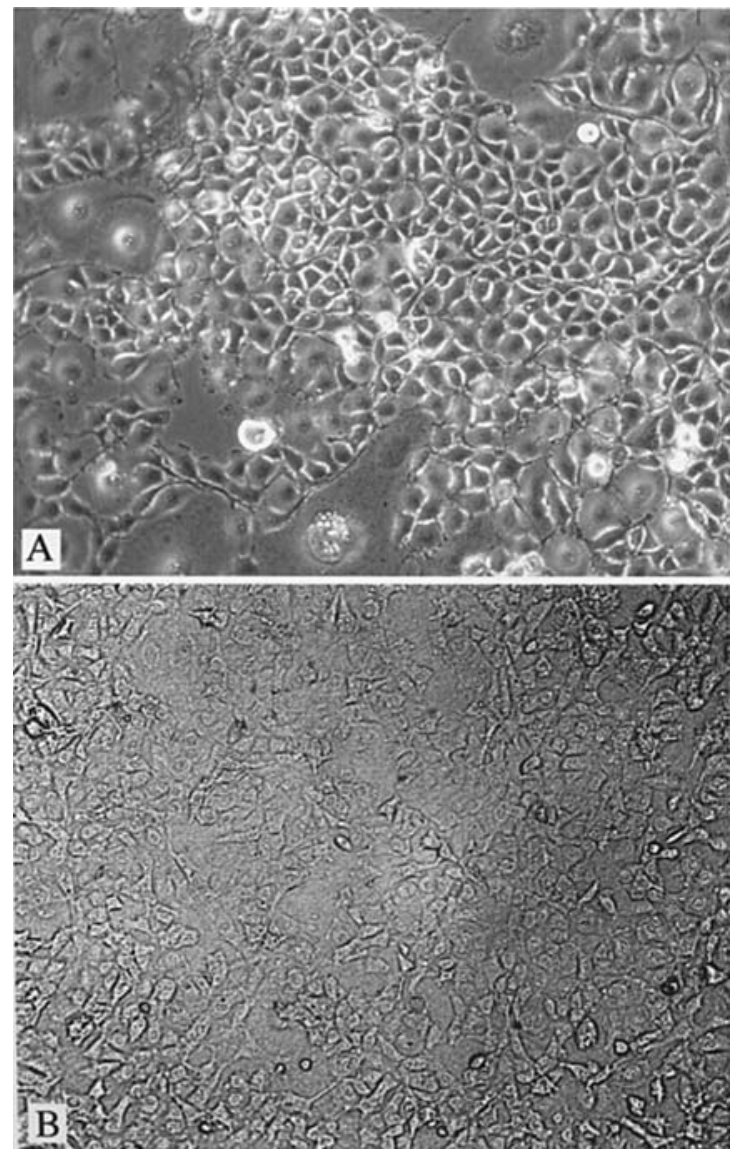

Figure 2. Morphology of ACCNS cells cultured on a plastic dish (A) and type I collagen gel (B). ACCNS cells consisted of small cuboidal cells and large spherical cells. When cultured on type I collagen gels, ACCNS cells showed epithelial characteristics with slender cytoplasmic processes. (Original magnification $\mathrm{x} 100$ ).

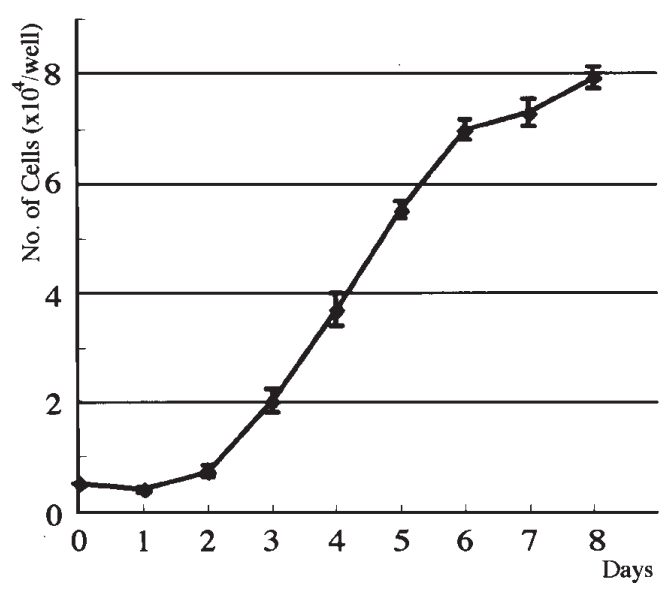

Figure 3. Cell growth curve of ACCNS. The viable cells were counted using the nigrosin exclusion test.

vacuoles in the cytoplasm and large nuclei. As shown in the cell growth curve, the population doubling time was approximately 39.2 h (Fig. 3).

$2 D$ culture on a plastic dish and collagen gels. ACCNS cells grew in a monolayer on the surface of both the plastic dish and collagen gels. When they were cultured on a plastic dish, 

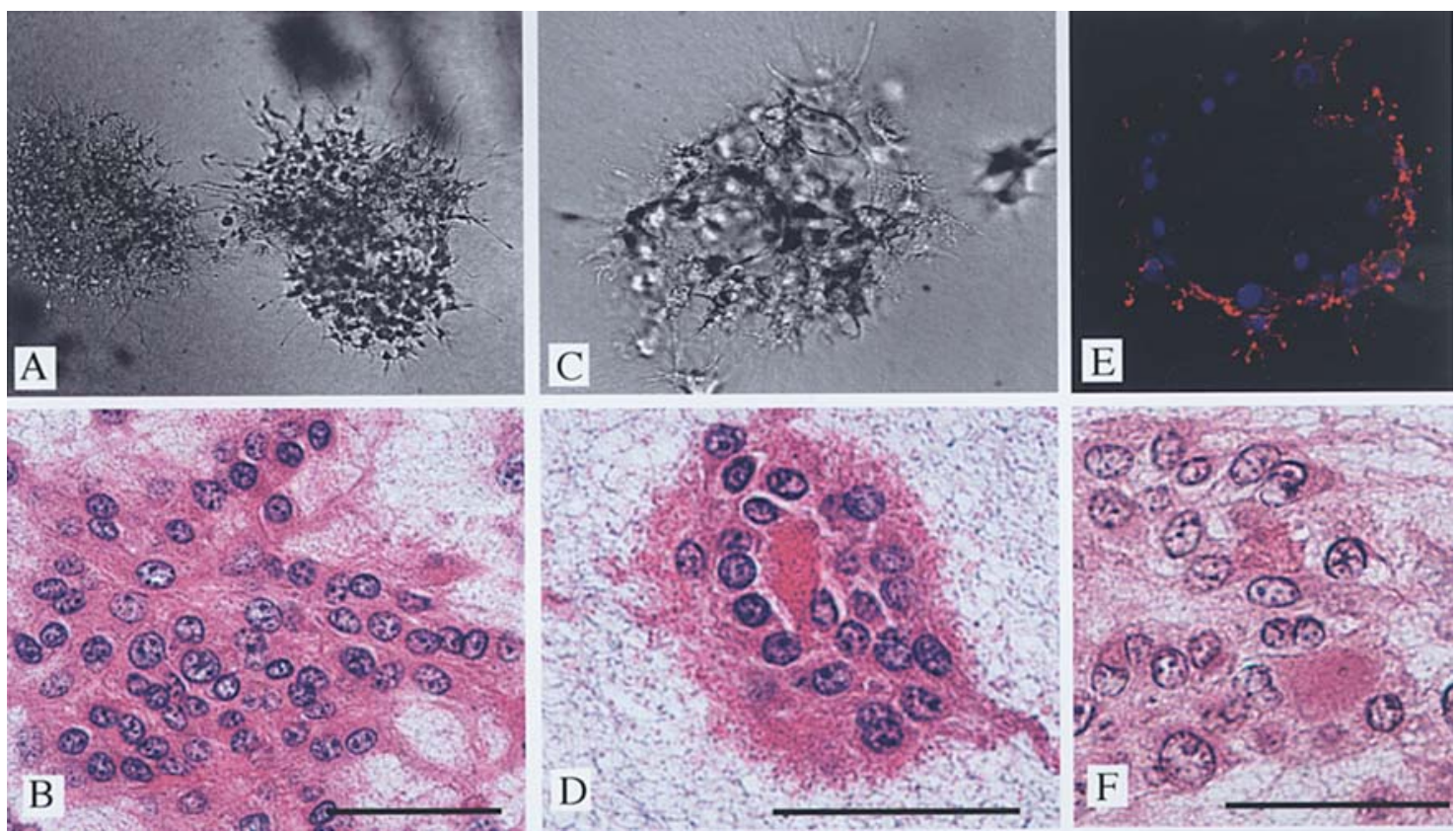

Figure 4. Morphological and histological features of ACCNS in collagen gel culture. Solid (A) and tubular (C) colony formation of ACCNS after 14 days in culture. [Original magnification (A), x100; (B), x200] H\&E stain of solid (C) and tubular (D) colony. Innner space of tubular colonies contained eosinophilic material (D). (H\&E stain; bar, $100 \mu \mathrm{m}$ ) Tubular space with immunofluorescence staining (E). (Red, actin; blue, nuclei; original magnification x480). Eosinophilic material in the inner space was positive with mucicarmine stain $(\mathrm{F})$. (Bar, $100 \mu \mathrm{m})$

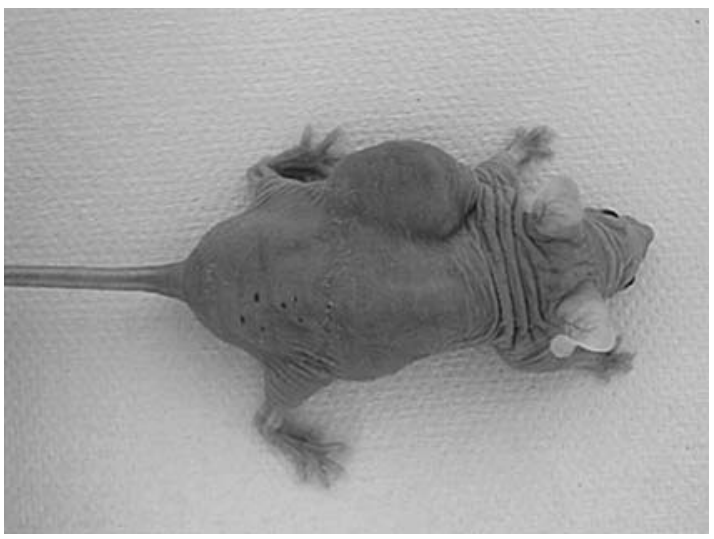

Figure 5. Subcutaneous tumor formed in nude mouse 3 months after cell inoculation.

they showed cuboidal and spherical cell shapes. On the other hand, when they were cultured on collagen gels, they showed cuboidal shape with slender cytoplasmic processes (Fig. 2B). However, the cells did not form any organoid structures in either culture condition.

$3 D$ culture in collagen gels. When cultured in collagen gels, ACCNS cells formed spherical colonies. All cells began to elongate slender processes into gel matrix $24 \mathrm{~h}$ after medium overlay. Within 7 days, these cells formed small colonies. By day 14 , some of the colonies grew to cystic, solid and tubular colonies (Fig. 4A and C). The edges of colonies were irregular and formed processes to contact with the peripheral cells. Histologically, the cells produced some colonies showing solid and tubular structures (Fig. 4B, D and E). Tubular colonies contained eosinophilic material in the inner space and it was positive with mucicarmine stain (Fig. 4F).
Tumorigenicity in nude mice. The tumorigenic potential of ACCNS cells was tested in 12 nude mice. Subcutaneous injection of $4.0 \times 10^{6}$ cells into the flanks of nude mice developed solid tumors 3 months later, approximately $25 \mathrm{~mm}$ in diameter, in two of 12 mice (Fig. 5). The tumor was encapsulated with fibrous connective tissues and the cut surface was pale-grayish in color. Histologically, the tumor was diffuse and showed non-cohesive proliferation of polyhedral epithelial cells with oval or round vesicular nuclei, prominent eosinophilic nucleoli and scant cytoplasm. These cells had indistinct cell margins and increased mitoses, corresponding to the characteristics of undifferentiated carcinoma (Fig. 1B). Adenoid structures were not observed in the tumors.

Immunohistochemical and immunofluorescence analysis. The immunohistochemical and immunofluorescence examinations revealed the similarity of cytoskeletal protein expressions between original tumor and ACCNS in $2 \mathrm{D}$ or $3 \mathrm{D}$ culture. Immunoreactivities of keratin, $\alpha$-SMA, vimentin and S-100 protein were detected in all three samples (Fig. 6A-L). EMA was positive in the original tumor but negative in ACCNS in 2D and 3D culture (Fig. 6M-O). Moreover, the inner space was observed in colonies of 3D culture (Fig. 4E).

Gene expression of human $\beta$-actin. RT-PCR analysis showed positive reaction with human $\beta$-actin and glyceraldehyde-3phosphate dehydrogenase (GAPDH) in ACCNS, HSG, SCC25 cells and nude mouse tumor produced by ACCNS, indicating that ACCNS is of human origin (Fig. 7).

\section{Discussion}

Establishment of AdCC cell lines has been reported (10-12), and two of these cell lines were cultured in $3 \mathrm{D}$ condition 


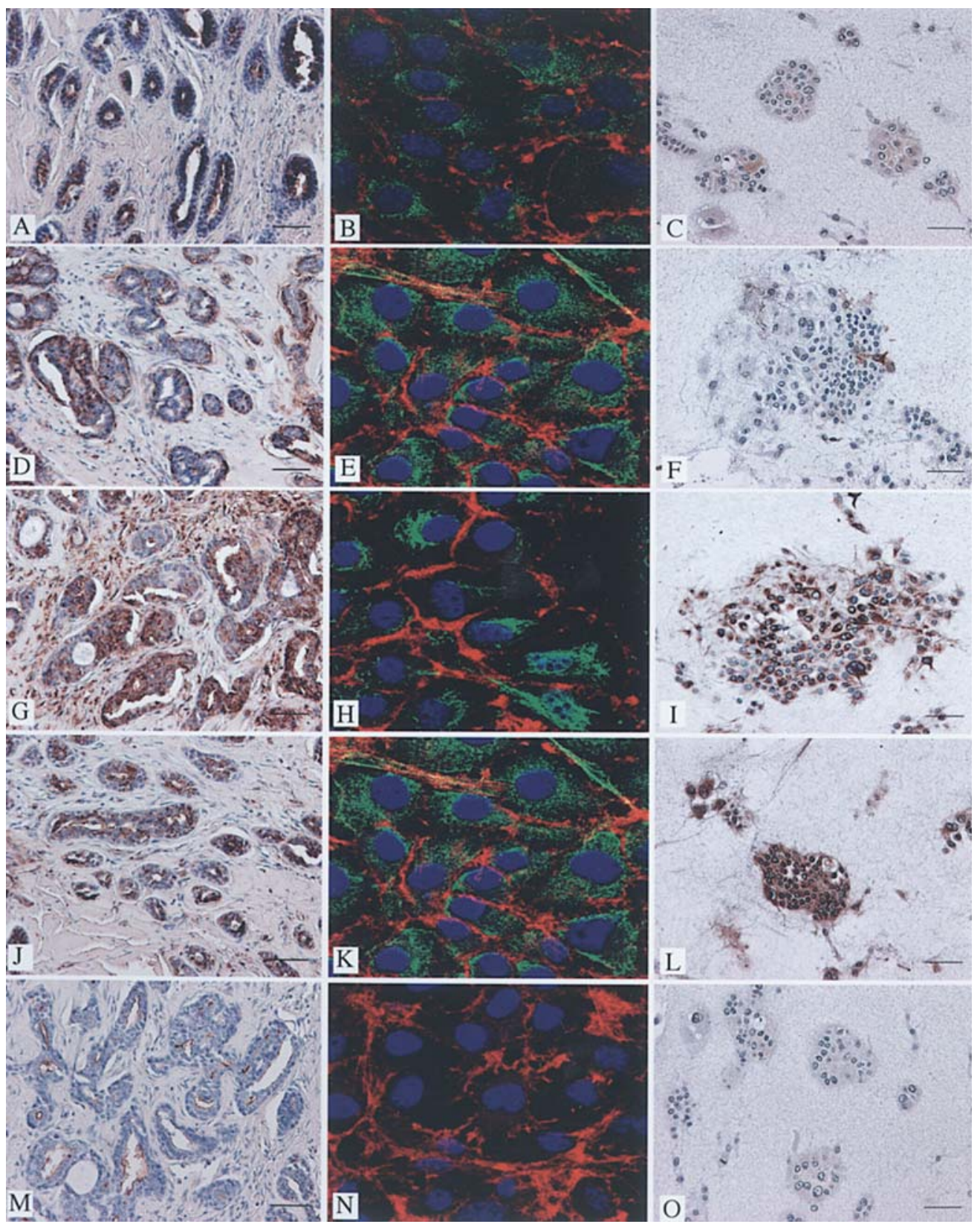

Figure 6. Immunohistochemical and immunofluorescent features of ACCNS and the original tumor. Keratin (A-C), $\alpha$-SMA (D-F), vimentin (G-I) and S-100 protein (J-L) showed positive reaction in the original tumor (A, D, G, J, M) and ACCNS in 2D (B, E, H, K, N) and 3D culture (C, F, I, L, O). EMA was positive in the original tumor $(\mathrm{M})$ but negative in ACCNS in $2 \mathrm{D}(\mathrm{N})$ and $3 \mathrm{D}(\mathrm{O})$ condition. (Bar, $100 \mu \mathrm{m}$, original magnification in immunofluorescence x400; red, actin; green, FITC-labeled secondary antibody; blue, nuclei).
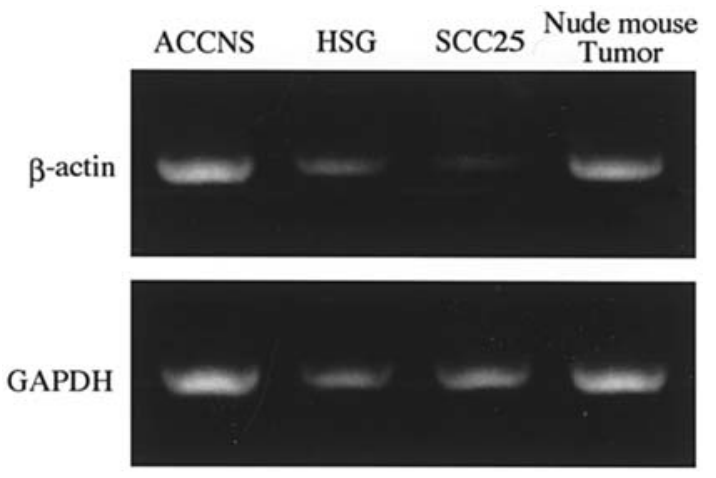

Figure 7. Detection of human B-actin mRNA in the ACCNS and nude mouse tumor by RT-PCR. Positive reaction is also seen in HSG and SCC25 cells. organized with solid agar (12). A recent study has described that epithelial tumor cells in 2D condition were different from $3 \mathrm{D}$ condition that is rich in type I collagen (biological condition) in cell shapes and cytoskeltal protein expression (13). Thus it is thought that since 3D condition in solid agar did not reflect the biological condition of AdCC, 3D culture condition in type I collagen gel is necessary to analyze biological behavior of AdCC. Only one previous study on culture in biological condition of AdCC cell line has been reported (5). In the present study, we established and characterized a new AdCC cell line (ACCNS) capable of being cultured in biological condition.

ACCNS was established from AdCC of the maxilla and possess high ability to grow in $2 \mathrm{D}$ condition. In $2 \mathrm{D}$ condition on a plastic dish, ACCNS cells showed monolayers and 
consisted of small cuboidal and large spherical cells. Histologically, AdCC is considered to arise from the terminal duct system (14); consisting of myoepithelial and intercalated duct cells (15). From the shape of ACCNS, it is speculated that small cuboidal cells arose from intercalated duct cells and large spherical cells arose from myoepithelial cells but the difference between cuboidal cells and spherical cells was not observed in expressions of cytoskeletal protein. In 2D condition on collagen gel culture, cells also showed monolayers and exhibited homogeneous cuboidal shape with slender processes. In biological condition of 3D collagen gels, cells formed spherical colonies with elongated cytoplasmic processes. Histologically, these colonies demonstrated tubular and solid patterns. In tubular pattern, eosinophilic material was found in the inner space and was positive with mucicarmine. However, cellular arrangement of cuboidal and spherical cells was not defined. These results suggested that it is important for colony formation of ACCNS to culture in $3 \mathrm{D}$ condition with type I collagen.

By immnohistochemistry, AdCC showned positive reaction with keratin and vimentin (16). In our study, original AdCC showed positive reaction with EMA, $\alpha$-SMA and S-100 protein as well as keratin and vimentin. Expression of keratin, vimentin, $\alpha$-SMA and S-100 protein was also demonstrated in 2D condition, but EMA was demonstrated in the original tumor but not in 2D and 3D culture conditions of ACCNS. Observation of these colonies in 3D culture condition with laser scanning microscope revealed the presence of inner space surrounding cuboidal cells with elongated slender processes in the center of the colonies. These inner spaces in colonies were considered as those compatible to cystic or tubular space of AdCC. These histological and immunohistochemical findings indicated that ACCNS was derived from AdCC and reflected the peculiarity of AdCC. Particularly, ACCNS cultured in 3D type I collagen gel expressed the representative behavior of AdCC.

ACCNS possessing the characteristics of AdCC maintained tumorigenicity in nude mice. ACCNS formed subcutaneous tumors in two of 12 nude mice inoculated with $4.0 \times 10^{6}$ cells. Previously, one cell line has been reported to produce tumors by subcutaneous inoculation into SCID mice (5), but there is no report of establishing a cell line having tumor forming ability in nude mice. Histologically, these tumors were diagnosed as undifferentiated carcinoma. From the result of RT-PCR, our nude mouse tumor expressed mRNA of human $B$-actin, indicating that this tumor was of human origin. The reason why AdCC changed into undifferentiated carcinoma is unclear and now under investigation.

In conclusion, ACCNS cell line is the first established cell line derived from AdCC being capable of formation of a cyst-like structure in 3D type I collagen gel culture and having tumorigenicity in nude mice. This cell line provides a useful model for investigating the biological behavior of AdCC.

\section{Acknowledgements}

This study was supported by a Grant-in-Aid for Scientific Research from the Ministry of Education, Sports, Science and Culture of Japan to N.T. (No. 18791535).

\section{References}

1. Hayashido Y, Yoshioka H, Tanaka N, et al: Dedifferentiation in adenoid cystic carcinoma of submaxillary gland: a case report. Oral Oncol Extra 41: 84-88, 2005.

2. Umeda M, Komatsubara H, Ojima Y, et al: Establishment and characterlization of metastasizing cell lines from a heterotransplanted human adenoid cystic carcinoma. Oral Surg Oral Med Oral Pathol Oral Radiol Endod 98: 211-216, 2004.

3. Hashitani S, Noguchi K, Manno Y, et al: Changes of histological and biological features by serial passages in human adenoid cystic carcinoma line transplantable in nude mice. Oncol Rep 13: 607-612, 2005.

4. Umeda M, Komatsubara H, Nishimatsu N, et al: Establishment and characterization of a human adenoid cystic carcinoma line of the salivary gland which is serially transplantable and spontaneously metastasis to the lung in nude mice. Oral Oncol 38: 30-34, 2002.

5. Munakata R, Irie T, Cheng J, et al: Pseudocyst formation by adenoid cystic carcinoma cells in collagen gel culture and in SCID mice. J Oral Pathol Med 25: 441-448, 1996.

6. Yang J, Richards J, Bowman P, et al: Sustained growth and three-dimensional organization of primary mammary tumor epithelial cells embedded in collagen gels. Proc Natl Acad Sci USA 76: 3401-3405, 1979.

7. Hsu SM, Raine L and Franger H: Use of avidin-biotinperoxidase complex (ABC) in immunoperoxidase techniques. A comparison between ABC and unlabeled antibody (PAP) procedures. J Histochem Cytochem 29: 577-580, 1981.

8. Shirasuna K, Sato M and Miyazaki T: A neoplastic epithelial duct cell line established from an irradiated human salivary gland. Cancer 48: 745-752, 1981.

9. Rheinwald JG and Beckett MA: Tumorigenic keratinocyte lines requiring anchorage and fibroblast support cultures from human squamous cell carcinomas. Cancer Res 41: 1657-1663, 1981.

10. He RG, Qiu WL and Zhou XJ: The establishment of Acc-2 and Acc-3 and their morphological observation. J West China Stromatol 6: 1-4, 1988.

11. Sobue M, Takeuchi J, Niwa M, et al: Establishment of a cell line producing basement membrane components from an adenoid cystic carcinoma of human salivary gland. Virchows Arch B Cell Pathol Include Mol Pathol 57: 203-208, 1989.

12. Shirasuna K, Watatani K, Furusawa $\mathrm{H}$, et al: Biological characterization of pseudocyst-forming cell lines from human adenoid cystic carcinomas of minor salivary gland origin. Cancer Res 50: 4139-4145, 1990

13. Hotary KB, Allen ED, Brooks P, et al: Membrane type I matrix metalloproteinase usurps tumor growth control imposed by threedimensional extracellular matrix. Cell 114: 33-45, 2003.

14. Seifert G, Miehlke A, Haubrich J and Chilla R: Disease of the salivary glands. Gerog Thieme Verlag, Stuttgart, pp239-248, 1986.

15. Neville BW, Damm DD, Allen CM and Bouquot JE: Oral and maxillofacial pathology. 2nd edition. W.B. Saunders Co., PA pp389-435, 2002.

16. Caselitz J, Becker J, Seifert G, et al: Coexpression of keratin and vimentin filaments in adenoid cystic carcinomas of salivary glands. Virchows Arch Pathol Anat 403: 337-344, 1984. 